\title{
Los talleres formativos: Reforzamiento y competitividad en la formación inicial IESPPA
}

\author{
Training workshops: Strengthening and competitiveness in initial training IESPPA
}

Silvia Quispe Flores, Alfredo Henry Laura Anco y Mario Alfredo Zevallos Luna

Instituto De Educación Superior Pedagógico Publico "Arequipa"

\section{INFORMACIÓN}

\section{Historia del Artículo}

Recepción: 06/04/2019

Revisión: 11/06/2019

Aceptación: 20/10/2019

\section{Palabras Clave}

Formación docente; talleres formativos; inteligencia emocional.

\section{Key Words}

Teacher training; training workshops; emotional intelligence

\section{DOI}

https://doi.org/10.35286/veritas. v21i1.255

\begin{abstract}
RESUMEN
La formación docente en la actualidad debe de responder a las necesidades del contexto, es decir, debe de estar preparado para afrontar la generación de jóvenes que tienen a la tecnología como un recurso que se usa desde diversas perspectivas y debe de introducir nuevas alternativas que permita también encaminar a esa generación a desarrollar potencialidades que no se pueden olvidar, si no por el contrario enseñarle a convivir con esos elementos, los talleres formativos apunta a desarrollar aquellas habilidades profesionales que la tecnología no puede atender cual es el contacto del calor humano, el desarrollo de la inteligencia emocional para replantear el sistema de vida en contacto con la naturaleza que necesita mucho de nosotros para ganar terreno en la relación con el hombre.
\end{abstract}

\begin{abstract}
Teacher training must now respond to the needs of the context, that is, it must be prepared to face the generation of young people who have technology as a resource that is used from different perspectives and should introduce new alternatives that allow also direct that generation to develop potentials that can not be forgotten, if not on the contrary to teach them to coexist with those elements, the training workshops aim to develop those professional skills that technology can not address which is the contact of human warmth, development of emotional intelligence to rethink the system of life in contact with nature that needs a lot of us to gain ground in the relationship with man.
\end{abstract}

\section{INTRODUCCIÓN}

Las Instituciones de Educación Superior que forman docentes tienen por misión mejorar la calidad en la formación que se debe de evidenciar en el desarrollo dela practica pedagógica y hacer del curriculo el macanismo que se va adaptando a las necesidades de las generaciones que estan en contacto con aquellas manifestciones de la tecnologia, del desarrollo social; de la representación de la convivencia compleja pero necesariapara comprender las causas que la generan. La preparacion a los estudiantes es constante, el termino calidad cada vez se va perfeccionando y creando nuevas necesidades, indicadores que permiten ir ajustando las estrategias para lograr los propósitos, retos de la formación inicial. Esos retos se refieren a que el conocimiento sea más integrado, científico ligado a lo práctico y que este en correspondencia con nuevos escenarios de ejercicio de la profesión, con nuevos perfiles y nuevas competencias. En casi todas las mallas curriculares se expresa de una u otra forma el propósito de la formación integral. En las "transformaciones" que se pretenden, muy pocas veces o ninguna, se reflexiona en el principal concepto que está en juego. Este concepto es el de formación.

Correspondencia:

Silvia Quispe Flores

silviadolores69@yahoo.es
Del análisis y profundización en ese término se precisó que su significado tiene una complejidad que se ignora y que por tal razón, no se toma en cuenta en las instituciones educativas como debe ser. La mayoría de estas IES se esmeran en proyectar un currículo para la formación de profesionales de una manera amplia y tratando de que no quede ningún deseo fuera. No obs $\neg$ tante, todo este esfuerzo todavía no alcanza a reflejar el proceso real de la formación de ese profesional, ni de la formación integral. Nuestro objetivo es evidenciar que los talleres formativos son de gran utilidad en el uso como metodología de fortalecimiento de competecias profesionales docentes.

Estas aproximaciones llevan a reflexionar cómo debe ser todo el proceso educativo de una Institución que forma futuros docentes para abarcar la formación y cuestionarse si el currículo, tal como se concibe, puede lograrla. De esta forma, surge también la pregunta de cuáles otros componentes curriculares pueden contribuir a realizar integralmente el proceso de formación.

Como es sabido por las investigaciones realizadas en los currículos, se ha constatado la existencia de cuatro tipos que conviven al mismo tiempo en ese proceso de formación, lo que muchas veces no es controlado. Está el currículo oficial (que es el que se elabora con varios do $\neg$ cumentos establecidos), el currículo vivido (es el proceso que realmente se realiza y que puede corresponder o no con el proyecto), el currículo oculto (que es el que no se proyecta pero está latente en él) y el currículo nulo (que aunque está proyectado no se realiza). 
En este escenario se puede establecer que implementar el proceso de formación es una tarea compleja que exige creatividad, responsabilidad y toma de decisiones acertadas con la participación integra de la comunidad educativa.

\section{El taller}

Ultimamente se han dado a conocer muchas definiciones sobre este espacio, para nosotros de carácter pedagógico por que nuestro campo es la de formar docentes los mas competitivos posibles en el mercado laboral magisterial, pero como se define, para Ander Egg "el taller reemplaza el mero hablar recapitulativo/repetitivo, por una hacer productivo en el que se aprende haciendo", (Egg, 1991) al margen de la formación que demanda el currículo de formación docente iniciamos una experiencia con esta propuesta que si bien cierto ya existía pero le damos un propósito para fortalecer las habilidades profesionales del futuro docente quien no solamente utilizará escenarios en el aula si no también fuera y además acompañado de un plus como actuar, dramatizar, danzar, usar las nuevas tecnologías, comprensión de idiomas; que por experiencia ayudo a nuestro estudiantes a ver su formación como una manifestación extrínseca en la especialidad que eligió como espacio de formación. Y por qué este investigador? Es quien nos manifiesta más clara esta afirmación, de él se valen los demás investigadores para tratar este tema, claro mejor parecer.

\section{Los talleres pedagógicos}

Por estar en campo educativo de todas maneras se necesita aportes pedagógicos para fortalecer el desarrollo de los talleres formativos donde "el taller pedagógico es un espacio en el que busca capacitar, orientar, investigar e intercambiar experiencias sobre un tema determinado; es un proceso de actualización como modalidad formativa, en el cual las acciones deben de estar destinadas a contribuir el desempeño del proceso Enseñanza- Aprendizaje" (Sanchez Morales, 2007) en este espacio fue fundamental juntar a estudiantes de diferentes programas académicos para compartir nuevas experiencias, la inscripción a los talleres fue con anticipación, donde los responsables hacían propaganda sobre el contenido del taller fue una experiencia enriquecedora porque se observó interacción entre los participantes de manera fluida, dinámica y sobre de inicio y fortalecimiento de la amistad. Es parte de la pedagogía interactuar por ser una generación donde los lazos de la amistad y lo académicos va a prevalecer en el tiempo y pronosticamos que se encontraran en el futuro para hacer más cosas en bien de la educación o ya algunos lo están haciendo en su comunidad.

\section{Los talleres formativos}

Es muy importante crear en los estudiantes los espacios para la reflexión sobre la formación docente donde el contexto demanda que éstos deben de asumir nuevas responsabilidades con los niños, adolescentes; padres de familia; directores, docentes y ser participe activo de los procesos de cambio donde se les designe la Práctica Pedagógica que es un espacio apropiado para recrear lo aprendido en los talleres formativos con sus aportes. En esta experiencia es el equipo encargado quien propone una malla curricular para el desarrollo de cada taller donde estos son amparados con una certificación por las horas óptimas asistidas siendo el jueves el día elegido para el desarrollo de lo planificado en el taller, pero como que alcances tenemos sobre ello, Gonzales Rivero, Berta nos manifiesta que "Es un componente organizativo del plan de estu $\neg$ dios, con una frecuencia específica, orientado hacia el desarrollo en los estudiantes de la capacidad crítica de su propio proceso de formación integral". (Gonzales Rivero, 2016) para participar de esta experiencia pasa por aprender algo nuevo, nuestros estudiantes asumen con responsabilidad su formación y es en este documento que Gonzales nos hace un aporte fundametal para ser tomado en cuenta en nesuras experiencias; por otro lado sabemos que tenemos que fortalecer cada taller ejecutado porque no fue facil concretizar varios procesos, el equipo identificó las fortalezas y tambien aquellos aspectos a superar como parte de la reflexión de la evaluación desarrollada que apunta al fortalecimiento de las competencia profesionales.

\section{La evaluacion de los talleres}

Partimos de una premisa que se esta haciendo cada vez mas contundente, cual es la evaluación formativa, una aproximación contundente nos hace Popham "la evaluación formativa es una herramienta potenciamente transformadora de la enseñanza que, si se ha entendido con claridad y se emplea adecuadamente puede beneficiar tanto a profesores como alumnos" (Popham, 2013) desde este punto podemos afirmar que los talleres formativos tiene este propósito la de hacer ver al estudiate y docente que puede incluir situaciones, innovaciones que impliquen asumir la docencia desde otra perspectiva la de docente critico reflexivo de los que hace dentro y fuera de los espacios educativos, po otro lado la escuela cunana nos dice que "es aquella que coadyuva al desarrollo del estudiante en correspondencia con las regularidades esenciales del proceso de formación del individuo y con las finalidades sociales que signan dicha formación en la sociedad. Además, es capaz de detectar los progresos y dificultades en el proceso enseñanza aprendizaje, determinar hasta dónde se ha llegado y hasta dónde se puede avanzar. Informa al estudiante de los hallazgos encontrados, lo que le permite al docente adecuar el currículo y los objetivos iniciales, y le otorgan la posibilidad de ajustar el proceso progresivamente" (Perez Pino, Enrique Clavero, , Carbó Ayala, \& Gonzáles Falcón , 2017) de ahí que los encargados de los talleres estan haciendo las reflexiones necearias para mejorar el servicio de la formación inicial entendiendo que se tiene que mejorar y sobre todo potencializar a los estudiantes en el desarrollo de las habilidades profesionales; los nuevos retos estan en camino la consigna, ser los mejores.

\section{Los recursos didácticos en los talleres formativos}

Desde el paradigma estratégico se tiene una visión que va con las intenciones del currículo sobre todo si se trata de los recursos que se emplearon en los talleres "Esta teoría crítica inspira un uso crítico y transformador de los medios, en los que éstos son utilizados como elementos de análisis y reflexión sobre la práctica incidiendo en la propia realidad con el fin de transformarla y mejorarla". (Bautista, 1994) Esta en relación con los que se pretende las actividades prácticas en los talleres requiere el apoyo de recursos que impliquen el desarrollo óptimo y sobre todo ahorrar el tiempo para desarrollar lo programado. Cada taller usó lo necesario de acuerdo a las exigencias de los formados y contundencia del docente encargado. 


\begin{tabular}{ccccc}
\hline & Frecuencia & Porcentaje & $\begin{array}{c}\text { Porcentaje } \\
\text { válido }\end{array}$ & $\begin{array}{c}\text { Porcentaje } \\
\text { acumulado }\end{array}$ \\
\hline $\begin{array}{c}\text { a) Tener expectativas } \\
\text { de aprendizaje como } \\
\text { futuro docente }\end{array}$ & 78 & 96,3 & 96,3 & 96,3 \\
$\begin{array}{c}\text { b) Para pasar el } \\
\text { tiempo }\end{array}$ & 3 & 3,7 & 3,7 & 100,0 \\
Total & 81 & 100,0 & 100,0 & \\
\hline
\end{tabular}

\section{Analisis e Interpretación}

En taller formativo es la metodología que ayuda a fortalecer las habilidades profesionales del futuro docente por su estructura y ejecución.

Como podemos observar que el $96,3 \%$ de los encuestados manifiestan que si tienen espectativa para fortalecer la formación profesional en los diferentes talleres elegidos

Por lo tanto si es muy importante esta propuesta desarrollada en el IESPPA.

Tabla 2. ¿Los profesores responsables para el taller formativo fueron los más indicados, cumplieron con los objetivos del taller?

\begin{tabular}{ccccc}
\hline & Frecuencia & Porcentaje & $\begin{array}{c}\text { Porcentaje } \\
\text { válido }\end{array}$ & $\begin{array}{c}\text { Porcentaje } \\
\text { acumulado }\end{array}$ \\
\hline $\mathrm{Si}$ & 60 & 74,1 & 74,1 & 74,1 \\
$\mathrm{No}$ & 21 & 25,9 & 25,9 & 100,0 \\
Total & 81 & 100,0 & 100,0 & \\
\hline
\end{tabular}

\section{Analisis e Interpretación}

El encargar a docentes y estudiantes cada taller implicó antes identificar, dialogar coordinar y responsabilizar cada uno de los talleres parala ejecición.

El $74,1 \%$ de estudiantes indican que si se logro los objetivos del taller que por cierto que todos expusieron el producto final, se lograron los objetivos previstos,

Los productos finales ayudaron mucho en la evaluación fina fue la unica manera de ver la efectividad del docente y la predisposición del estudiaste para aprender.

Tabla 3. ¿El sistema de evaluación por desempeño empleado fue el más indicado?

\begin{tabular}{ccccc}
\hline & Frecuencia & Porcentaje & $\begin{array}{c}\text { Porcentaje } \\
\text { válido }\end{array}$ & $\begin{array}{c}\text { Porcentaje } \\
\text { acumulado }\end{array}$ \\
\hline $\mathrm{Si}$ & 57 & 70,4 & 70,4 & 70,4 \\
$\mathrm{No}$ & 24 & 29,6 & 29,6 & 100,0 \\
Total & 81 & 100,0 & 100,0 & \\
\hline
\end{tabular}

\section{Analisis e Interpretación}

La evaluación formativa fue mas humana poque se entendió que tanto docentes y estudiantes participaban del proceso de transformación y que se hiba de manos a mas.

El $70,4 \%$ de los estudiantes manifiestan que si la evaluación fue por subproductos y producto final donde en dias determinados y para todos los estudianes demostraron lo aprendido; y por otro lado habia que reflexionar sobre aquellos aspectos a mejorar.
Fue muy importante evaluar por que permitio verificar los procesos plainificados y desarrollados.

Table 4. ¿La metodología de enseñanza- aprendizaje empleada fue las adecuada?

\begin{tabular}{ccccc}
\hline & Frecuencia & Porcentaje & $\begin{array}{c}\text { Porcentaje } \\
\text { válido }\end{array}$ & $\begin{array}{c}\text { Porcentaje } \\
\text { acumulado }\end{array}$ \\
\hline $\mathrm{Si}$ & 69 & 85,2 & 85,2 & 85,2 \\
$\mathrm{No}$ & 12 & 14,8 & 14,8 & 100,0 \\
Total & 81 & 100,0 & 100,0 & \\
\hline
\end{tabular}

\section{Analisis e Interpretación}

La metodología de enseñanza y aorendizaje es el conjunto de acciones que cada docente asumio en los diferentes talleres de acuerdo a la caracteristicas de ellos; todas furon de reflexión cosntante sobre el apendizaje y el dcente sobre la enseñanza.

El $85,2 \%$ indica que la metodologi de enseñanza y aprendizaje fue la mas adecuada por la participación activa que tuvieron, el lenguaje empleado, las consignas dadas, el tiempo empleado, y sobre todo la claridad de los objetivos propuestos de cada taller.

Finalmente, los participantes del los talleres si estbieron a gustos con la metodologia empleada.

Table 5. Los recursos, materiales empleados para desarrollar los fundamentos teóricos, práctica de actividades artísticas, deportivas, recreativas en el taller fueron:

\begin{tabular}{ccccc}
\hline & Frecuencia & Porcentaje & $\begin{array}{c}\text { Porcentaje } \\
\text { válido }\end{array}$ & $\begin{array}{c}\text { Porcentaje } \\
\text { acumulado }\end{array}$ \\
\hline $\begin{array}{c}\text { Los más } \\
\text { adecuados }\end{array}$ & 48 & 59,3 & 59,3 & 59,3 \\
$\begin{array}{c}\text { Había ciertas } \\
\text { imprecisiones }\end{array}$ & 24 & 29,6 & 29,6 & 88,9 \\
$\begin{array}{c}\text { De vez en } \\
\text { cuando se } \\
\text { utilizaba }\end{array}$ & 6 & 7,4 & 7,4 & 96,3 \\
$\begin{array}{c}\text { No se utilizaba } \\
\text { Total }\end{array}$ & 3 & 3,7 & 3,7 & 100,0 \\
\hline
\end{tabular}

\section{Analisis e Interpretación}

En el desarrollo de un taller implica el uso de recursos que son los medios que ayudan a concretizar los propósitos establecidos, es una ayuda que el docente responsable utiliza para mejorar su actuar y sobre todo la del estudiante.

El 59,3\% indican que fueron los mas adecuados debido a que ayudo a comprender, desarrllar la ejecusión de los ejerciciós y otros planificados por el responsable; mientra que el $88,9 \%$ manifiesta que hubo ciertas imorecisiones puede que se deba a que recien se estuvieron familiarizando con los talleres, un bue sector de estudiantes no conocian el taller y comenzaron de 0 .

Los recursos fueron de gran ayuda para el desarrollo de cada uno de los talleres. 


\section{CONCLUSIONES}

La organización y ejecución de los talleres formativos ayuda a los estudiantes de formación inicial a fortalecer las competencias profesionales, fue una decisión acertada por el equipo directivo jerárquico, docentes y de estudiantes; los paradigmas y enfoques de una educación transformadora ayuda a tomar decisiones acertadas que si bien es cierto cuestan pero se debe de asumir con responsabilidad porque ayudan a fortalecer la visión y misión de nuestra institución. Estamos en camino de ser referentes en la formación inicial docente en nuestra región

\section{REFERENCIAS BIBLIOGRÁFICAS}

1. Bautista, A. (1994). Las nuevas tecnologías en la capacitación docente. Madrid: VISOR.

2. Egg, E. A. (1991). Taller una Alternativa de la Renovacion Pedagogica. Buenos Aires: Magisterio del Rio De la Plata.

3. Gonzales Rivero, B. M. (2016). El taller como componente curricular para la formación integral en la educación superior. Perspectivas docentes, 9.

4. Perez Pino, M., Enrique Clavero, , j., Carbó Ayala, J., \& Gonzáles Falcón , M. (2017). La evaluación Formativa en el procesode enseñanza aprendizje. EDUMECENTRO, 97.

5. Popham, J. W. (2013). La evaluación trans-formativa. España: Narcea.

6. Sanchez Morales, C. E. (2007). El taller Pedagógico una alternativa de actualización. Zamora: UPN-E. 